\title{
-NOTES-
}

\section{ON THE WORK OF A FORCE CROSSING A BEAM *}

BY L. MAUNDER (University of Edinburgh)

1. Introduction. Timoshenko [1] has discussed the paradox that during its transit across an elastic beam whose ends are supported at the same horizontal level, as shown in Fig. 1, a vertical force apparently performs no net work, yet leaves the beam in a state of oscillation. His explanation, however, refers to a different but closely related problem, in which the force is always directed at right-angles to the deflected beam. A horizontal force component is thereby introduced which evidently is capable of doing work.

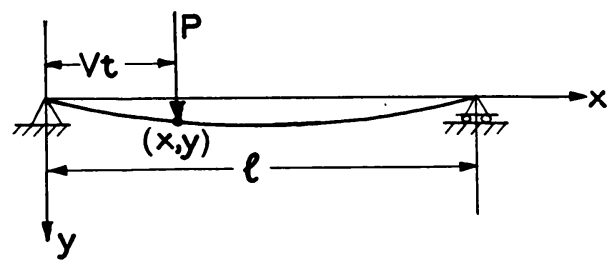

FIG. 1

Lee [2] shows that this work equals the sum of the strain and kinetic energies possessed by the beam on departure of the force. But, pointing out that this result does not explain the original difficulty, he returns to the case of the purely vertical force and shows that, contrary to appearances, the work which it does on the beam is not zero, but equals the energy retained by the beam. This result then resolves, in principle, the original paradox.

In view of the usual interpretation of the work of a force, however, it remains curious that the purely vertical force does positive work during transit between points on the same horizontal level. It therefore seemed worthwhile to consider the problem further, not only because of the physical mechanisms involved, but also because of its bearing on the definition of work.

2. Work relations. As in [2], we define the rate at which a force does work on a body as the scalar product of the force vector and the velocity vector of the particle of the body at the point of application of the force. This definition permits association of the work done on the body with the change in its kinetic, strain and potential energy. But it does not always describe fully the transmission of work from one physical system to another, as may be illustrated by the system of Fig. 2 .

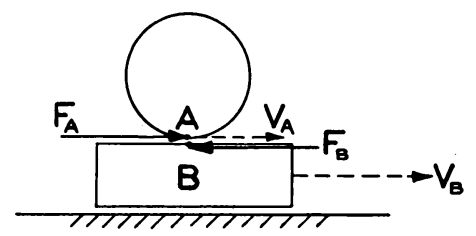

Frg. 2

*Received July 30, 1958; revised manuscript received October 27, 1958. 
Block $B$ slides on a frictionless horizontal plane with velocity $V_{B}$ : the center of the circular disc moves horizontally only and the disc rotates such that the velocity of particle $A$ in contact with the block is $V_{A}$. As the normal components of the contact forces between disc and block do no work, only the frictional components $F_{A}$ and $F_{B}$ are shown. From the preceding definition we find that $F_{B}$ does work on the block at the rate $\left(-F_{B} V_{B}\right)$ and that $F_{A}$ does work on the disc at the rate $\left(F_{A} V_{A}\right)$. This description can usefully be expanded, however, by stating that $F_{B}$ extracts work from the block at the rate $\left(F_{B} V_{B}\right)$, of which part is transmitted to the disc at the rate $\left(F_{A} V_{A}\right)$ and part is transformed into heat by the force pair $F\left(F_{A}=F_{B}=F\right)$ at the rate $F\left(V_{B}-V_{A}\right)$.

If $V_{A}$ is zero, when the figure might represent metal-cutting with a fixed tool at $A$, work is extracted by the contact force from the traveling work-piece $B$ at the rate $\left(F V_{B}\right)$. All of the extracted work is transformed by the contact force pair and no work is done on the fixed tool. If $V_{A}=V_{B}=V$, so that the disc rolls on the block, work is extracted by the contact force from one body at the rate $(F V)$ and is transferred entirely to the other. In this case, no work is transformed into heat.

In general the flow of work from one system to another should be studied through the action of the force pair or pairs exerted between the systems. When tangential forces occur at moving surfaces, the foregoing definition of work can lead to the result that the work extracted from one system differs from that transmitted to the other. In certain cases, the difference is transformed into energy associated with microscopic phenomena, such as thermal energy.

3. The physical system. The application of a purely vertical force to the deflected beam requires that the surface of the beam be capable of sustaining tangential forces. They may be associated with sliding or rolling. We consider both possibilities.

A particle of negligible mass can slide across the beam in the prescribed way under the action of an applied constant vertical force and the equal and opposite reaction of the beam, assuming that the frictional properties of the surfaces allow the latter to be developed. The total work done on the particle by the applied force is expressed by the integral

$$
P \int_{0}^{l / V}\left(\frac{\partial y}{\partial t}+V \frac{\partial y}{\partial x}\right)_{x=V t} d t
$$

in which the integrand is the vertical component of the velocity of the particle. As the net vertical displacement of the particle is zero, the work (1) is zero. Since the particle stores no work, the total work extracted from it by the contact force applied by the beam must also be zero. Now the (zero) extracted work is partly transferred to the beam and partly transformed into heat by the frictional components of the contact force pair. The net work done on the beam is expressed [2] by the integral

$$
P \int_{0}^{l / v}\left(\frac{\partial y}{\partial t}\right)_{x=v t} d t
$$

which, being equal to the energy retained by the beam, is necessarily positive. Hence the work transformed into heat, namely,

$$
P V \int_{0}^{l / v}\left(\frac{\partial y}{\partial x}\right)_{x=V t} d t
$$

must be negative, from which we conclude that the assumed frictional characteristics 
cannot exist even in principle. The detailed explanation is that the assumptions entail negative friction, i.e. friction tending to increase the relative velocity between particle and beam, when $(\partial y / \partial x)_{x=v t}$ is negative. Sliding friction must therefore be discarded as a possibility.

The alternative is rolling contact, of which the simplest case is the rolling of a circular disc of negligible mass and radius $r$. Its point of contact with the beam will have the prescribed horizontal velocity $V$ if the velocity of its center has horizontal and vertical components $V$ and $[(\partial y / \partial t)+V(\partial y / \partial x)]_{x=V t}$ respectively, and if the disc has a clockwise angular velocity

$$
(V / r)\left[1+(\partial y / \partial x)^{2}\right]_{x=V t}^{1 / 2} .
$$

This motion requires that certain forces be applied to the disc in addition to the prescribed vertical reaction $P$ of the beam. They are equivalent to a vertical force $P$ applied at the center of the disc together with a counterclockwise couple of magnitude $\operatorname{Pr}\left\{(\partial y / \partial x) /\left[1+(\partial y / \partial x)^{2}\right]^{1 / 2}\right\}_{x=v t}$.

As the disc stores no work, the work done on it by the applied central force and the applied couple must be extracted fully by the contact force of the beam. Moreover, the work so extracted is transferred entirely to the beam, since no work is transformed at a rolling contact, as noted previously. Now the applied central force and the applicd couple do work on the disc at the rates

$$
P[(\partial y / \partial t)+V(\partial y / \partial x)]_{x=v} \text { and }-P V(\partial y / \partial x)_{x=V t}
$$

respectively, as follows from definition and the prescribed motion of the disc. Hence the rate at which work is transferred by the contact force to the beam is the sum of the rates (4), namely,

$$
P(\partial y / \partial t)_{x=v t} .
$$

If we regard the central vertical force and the couple as external forces which are applied to the system of disc and beam, it is noteworthy that the total work done on the system by the central force is zero. The work done on the system by the couple equals the work done on the beam by the contact force, i.e.

$$
-P V \int_{0}^{l / v}\left(\frac{\partial y}{\partial x}\right)_{x=v t} d t=P \int_{0}^{l / v}\left(\frac{\partial y}{\partial t}\right)_{x=V t} d t .
$$

4. Conclusion. A full description of the work of a force requires specification of the physical nature of the systems concerned. A physical inconsistency appears if the vertical force is considered to be applied to the beam through a sliding body, but it can be applied through a rolling body. In the simplest case of a rolling circular disc of negligible mass, the external forces which must be applied to the disc are equivalent to a constant vertical force applied at the center of the disc and a couple. The vertical force does no net work on the disc during transit of the beam, and the couple does an amount of work on the disc equal to that retained by the beam.

\section{REFERENCES}

1. S. Timoshenko, Vibration problems in engineering, Van Nostrand, New York, 1937, p. 355

2. E. H. Lee, On a "paradox" in beam vibration theory, Quart. Appl. Math. 10, pp. 290-292 (1952) 